\section{Case Reports in Ophthalmology}

\begin{tabular}{l}
\hline Case Rep Ophthalmol 2013;4:138-143 \\
\hline \begin{tabular}{l|l} 
DOI: 10.1159/000355415 & $\begin{array}{l}\text { ○ 2013 S. Karger AG, Basel } \\
\text { 1663-2699/13/0043-0138 } \$ 38.00 / 0 \\
\text { www.karger.com/cop }\end{array}$ \\
\hline
\end{tabular}
\end{tabular}

This is an Open Access article licensed under the terms of the Creative Commons Attribution-NonCommercial 3.0 Unported license (CC BY-NC) (www.karger.com/OAlicense), applicable to the online version of the article only. Distribution permitted for noncommercial purposes only.

\title{
Reverse Optic Capture to Stabilize a Toric Intraocular Lens
}

\author{
Howard V. Gimbel ${ }^{a, b} \quad$ Anika Amritanand ${ }^{a}$

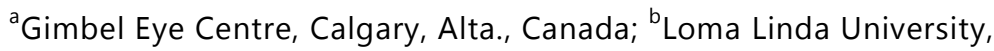 \\ Loma Linda, Calif., USA
}

\section{Key Words}

Toric intraocular lens $\cdot$ Rotation $\cdot$ Reverse optic capture

\begin{abstract}
Purpose: To describe a technique for stabilizing a rotationally unstable toric intraocular lens (IOL). Method: Case report and literature review. Results: Surgical technique and long-term follow-up for a patient who underwent repositioning and stabilization of a mobile 1-piece acrylic toric IOL using reverse optic capture (ROC) are described. This patient presented with early, more than $70^{\circ}$ off-axis rotation. The IOL was repositioned but was very mobile within the bag and tended to rotate off-axis; hence, it was stabilized in the desired position by capturing the optic through the anterior continuous curvilinear capsulorhexis, leaving the haptics in the bag. The immediate and 2-year postoperative follow-up revealed a stable and on-axis IOL with no visual, refractive or ocular complications. Conclusions: ROC is a useful and safe technique to address the problem of toric IOLs that tend to rotate at the time of surgery or are not stable postoperatively.

(c) 2013 S. Karger AG, Basel
\end{abstract}

\section{Introduction}

Toric intraocular lenses (IOLs) are a recognized and proven treatment for correcting preexisting corneal astigmatism in patients undergoing cataract surgery [1]. However, even small degrees of misalignment of the toric IOL away from the correct axis of implantation can adversely affect the refractive effect [2]. Fortunately, this complication is rare, and when significant rotation is present it can be treated easily with repositioning [3].

There are, however, instances in clinical practice when toric lenses rotate a large and unacceptable amount from the correct axis, are not stable with an early repositioning 
Gimbel et al.: Reverse Optic Capture to Stabilize a Toric Intraocular Lens

procedure and may require repeated repositioning [4,5]. We describe 1 such patient who underwent reverse optic capture (ROC) of an unstable toric IOL with excellent results.

\section{Case Report}

A 38-year-old woman, with no known cause for premature cataract apart from a family history of early cataract surgery, underwent cataract extraction in the left eye with implantation of a 1-piece acrylic toric IOL (SN6AT4; Alcon Laboratories, Inc.), with a power of +17 D sphere, 2.25 D (IOL plane), and $1.55 \mathrm{D}$ (corneal plane) toric power. She had undergone cataract surgery with spherical foldable IOL implantation in the right eye 1 year before. Apart from an immature cataract in the left eye, the rest of the ocular examination was unremarkable.

The preoperative manifest refraction in the left eye was $-0.75-1.25 \times 148^{\circ}$. Her preoperative axial length was $24.8 \mathrm{~mm}$ and her keratometry values were $42.99 @ 158^{\circ}$ and $44.76 @ 68^{\circ}(1.77 \mathrm{D}$ cyl $)$ as measured by the IOL Master. According to the Acrysof IQ IOL calculator (www.acrysoftoriccalculator.com/), the recommended axis of implantation was $64^{\circ}$ using a superior incision with zero-induced cylinder.

Preoperatively, the horizontal axis was marked by the surgeon using a fine-tipped sterile marking pen under topical anesthesia (Surgeons marking pen 115; Medical Action Industries, Inc.). The marking was made at the 3 and 9 o' clock positions on the limbus with the patient seated at the slit lamp, ensuring the 2 eyes were level with no head tilt. The $64^{\circ}$ axis was marked under the operating microscope using the Cionni Reverse Bevel Mendez Fixation Ring (Mastel Precision). She underwent phacoemulsification under topical anesthesia using the chop divide and conquer technique through a superior conjunctiva sparing scleral tunnel incision [6]. A toric IOL was implanted with the axis aligned to $64^{\circ}$. After all the viscoelastic was aspirated the anterior chamber was reformed with balanced salt solution (BSS; Alcon Laboratories, Inc.), the wound and the paracentesis were hydrated, and the intraocular pressure brought to normal. The IOL was confirmed again to be at the $64^{\circ}$ axis.

On the first postoperative day, the patient's uncorrected visual acuity was $20 / 70$ with a manifest refraction of $+0.75-2.25 \times 143^{\circ}$. The axis of IOL rotation was measured with pupils dilated using the slit lamp method with a thin slit beam aligned along the axis markings of the IOL, and also with an internal OPD map on the OPD-Scan refractive power/corneal analyzer system (Nidek). The axis of the IOL had rotated to $135^{\circ}$. The dilated OPD-Scan refraction showed an internal astigmatism of -1.41 at $120^{\circ}$. (fig. 1). Because of the large degree of IOL rotation and refractive error, it was decided to not wait for capsule contraction before surgically repositioning the IOL. The repositioning procedure was done on the fifth day postoperatively.

\section{Surgical Technique}

The patient consented to toric IOL repositioning. The horizontal axis and the $64^{\circ}$ axis were marked as described earlier. The left eye was prepared and draped with all sterile precautions. Under topical anesthesia, the limbal paracentesis was reopened and intracameral nonpreserved lidocaine $1 \%$ and phenylephrine $2.5 \%$ (8:1) ratio was instilled intracamerally. The axis of the IOL had rotated to $130^{\circ}$. The BSS cannula was used to rotate it to the $64^{\circ}$ position. The IOL was found to be very loose in the bag and tended to rotate again 
Gimbel et al.: Reverse Optic Capture to Stabilize a Toric Intraocular Lens

during irrigation of the BSS to bring the IOP to normal. Therefore, it was decided to proceed with stabilizing the IOL using ROC. The axis markings on the IOL were aligned to the $64^{\circ}$ axis, a second paracentesis was formed opposite to the existing one and 1 edge of the IOL was brought out of the continuous curvilinear capsulorhexis (CCC) edge with a Sinsky hook $90^{\circ}$ from the axis markings, while the optic was stabilized with a spatula. After ensuring alignment, the other edge of the IOL was also brought anterior to the CCC edge with the Sinsky hook $90^{\circ}$ from the axis markings, thus capturing the IOL in the desired position. The paracentesis was hydrated and the patient was taken to the recovery room in good condition.

On the first postoperative day after repositioning with ROC, the patient's unaided vision was 20/20. The optic was well captured in front of the CCC and was stable at the axis of placement (fig. 2). At the 2 -week postoperative visit, the manifest refraction was $-0.75+0.25$ $\times 150^{\circ}$.

At the 2-year follow-up after ROC, the patient was not using any correction for her distance vision, unaided vision was $20 / 20$, her manifest refraction was plano $-0.25 \times 97^{\circ}$, and the pupils were equal and briskly reactive. The IOL was captured by the CCC at the axis of $65^{\circ}$. Her intraocular pressure was $14 \mathrm{~mm} \mathrm{Hg}$ in the right eye and $13 \mathrm{~mm} \mathrm{Hg}$ in the left eye. There was no evidence of any pigment dispersion or iris chaffing. The posterior segment examination was normal.

\section{Discussion}

ROC, a technique that captures the optic of an IOL above the CCC with the haptics remaining in the bag, has been described in the literature as an option for safe implantation of a posterior chamber IOL in the presence of a large posterior capsule tear, radial extension of a primary posterior CCC and to fixate piggyback IOLs [7]. It has also been used to correct mild postoperative hyperopia in patients who have undergone multifocal IOL implantation [8]. More recently, it has been successfully used to eliminate negative dysphotopsia in pseudophakic patients and also as part of combined cataract and vitreoretinal surgery [9, 10]. To the best of our knowledge, this is the first time in the literature that this method is being described to stabilize a toric IOL that is mobile within the bag.

In the event that a toric IOL does not stabilize by a routine repositioning or is felt to be loose and mobile within the bag, ROC is a simple and safe option. Toric IOL rotation has been positively correlated with longer axial lengths and, consequently, large capsular bags [3]. Our patient's axial length $(24.8 \mathrm{~mm})$, and white-to-white diameter $(12.4 \mathrm{~mm})$ were on the higher side of normal, and we found that she had a large capsular bag within which the lens was tending to rotate freely.

One option to treat this case would have been to wait for a period of a few weeks until capsular fibrosis reduced the diameter of the capsular bag to ensure stability of the IOL after repositioning. This would, however, require a waiting period with poor vision. Another option in this patient would have been a corneal laser procedure to correct the astigmatism, but this would not solve the problem of a rotating lens without a waiting period until capsular fibrosis stabilized the IOL. ROC can be done simply and quickly, not requiring any more time or instrumentation than for a routine surgical repositioning. The prerequisites for ROC are an intact, central CCC that is slightly smaller than the optic diameter, which is the ideal size of the CCC that most surgeons aim for.

There have been concerns regarding the complications due to the forward position of the IOL and whether this would lead to complications similar to those with sulcus-fixated 
Gimbel et al.: Reverse Optic Capture to Stabilize a Toric Intraocular Lens

IOLs. We believe that the dynamics of an IOL captured in front of and by the CCC are very different from that of a sulcus-placed IOL. The captured IOL is fixed and immobile with the haptics still within the bag; hence, there is little chance of iris chaffing or uveitis-glaucomahyphema syndrome. Ultrasound biomicroscopy pictures of patients with ROC do not show excessive touch between the IOL and the posterior surface of the iris in pseudophakic eyes [9]. Akaishi et al. [8] reported 1 case of glaucoma out of 16 patients who had undergone ROC, but mechanics related to ROC could not be confirmed by ultrasound biomicroscopy.

The change in the refractive status of the lens needs to be considered, as one would expect a myopic change due to the forward movement of the optic. Akaishi et al. [8] reported a $0.81 \mathrm{D}$ of myopic change. In our patient, the spherical equivalent was $-0.625 \mathrm{D}$ at 2 weeks postoperatively, and this reduced to $-0.12 \mathrm{D}$ at 2 years, probably due to capsular retraction causing the lens to move backward. The preoperative predicted spherical equivalent with this lens power was -0.15 D. Hence, we recommend no adjustment of calculation in lens power for these patients. Though there have been no long-term studies on refractive change due to ROC, other authors utilizing this procedure for other indications also suggest the same $[10,11]$.

The disadvantages of this procedure are that it cannot be done in the presence of a CCC that is decentered or larger than the optic diameter. Zonular weakness would also be a contraindication to using this procedure. Change in the refractive error due to lens position should be kept in mind; therefore, this procedure may not be ideal for eyes with short axial lengths and high IOL powers, unless a myopic shift is desired. However, this does not factor in as much in eyes with a low IOL power and long axial lengths, the very type of eyes which may have larger bags and, hence, rotationally unstable IOLs.

\section{Conclusion}

We describe a simple, safe and cost-effective method to manage rotationally unstable toric IOLs which may be recalcitrant to an early, simple repositioning procedure.

\section{Disclosure Statement}

The authors have no proprietary or financial interest concerning the method, products or instruments described. The authors did not receive any financial support from any public or private sources. This article has not been presented at a meeting.

\section{References}

1 Visser N, Bauer NJ, Nuijts RM: Toric intraocular lenses: historical overview, patient selection, IOL calculation, surgical techniques, clinical outcomes, and complications. J Cataract Refract Surg 2013;39:624637.

2 Ma JJ, Tseng SS: Simple method for accurate alignment in toric phakic and aphakic intraocular lens implantation. J Cataract Refract Surg 2008;34:1631-1636.

3 Chang DF: Repositioning technique and rate for toric intraocular lenses. J Cataract Refract Surg 2009;35:1315-1316.

4 Safran SG: Taking the spin out of toric rotation: part 2. Eye World 2013;18:10-11.

5 Safran SG: Taking the spin out of toric rotation: part 1. Eye World 2012;17:12-13.

6 Gimbel HV, da Reitz Pereira C, Niemeyer MS: Conjunctival Advancing Scleral Tunnel (CAST) incision. Clin Surg Ophthalmol 2009;27:54-58.

7 Gimbel HV, DeBroff BM: Intraocular lens optic capture. J Cataract Refract Surg 2004;30:200-206. 


\begin{tabular}{l|l}
\hline DOI: 10.1159/000355415 & $\begin{array}{l}\text { ○ } 2013 \text { S. Karger AG, Basel } \\
\text { www.karger.com/cop }\end{array}$ \\
\hline
\end{tabular}

Gimbel et al.: Reverse Optic Capture to Stabilize a Toric Intraocular Lens

8 Akaishi L, Bessa T, Vaz R, Canamary F, Tzelikis PF: Multifocal intraocular lens optic anteriorization capture to correct residual refractive error. J Cataract Refract Surg 2009;35:2077-2083.

$\checkmark 9$ Masket S, Fram NR: Pseudophakic negative dysphotopsia: surgical management and new theory of etiology. J Cataract Refract Surg 2011;37:1199-1207.

10 Lee JE, Ahn JH, Kim WS, Jea SY: Optic capture in the anterior capsulorhexis during combined cataract and vitreoretinal surgery. J Cataract Refract Surg 2010;36:1449-1452.

11 Carifi G: Capsulorhexis optic intraocular lens capture technique. J Cataract Refract Surg 2011;37:427.

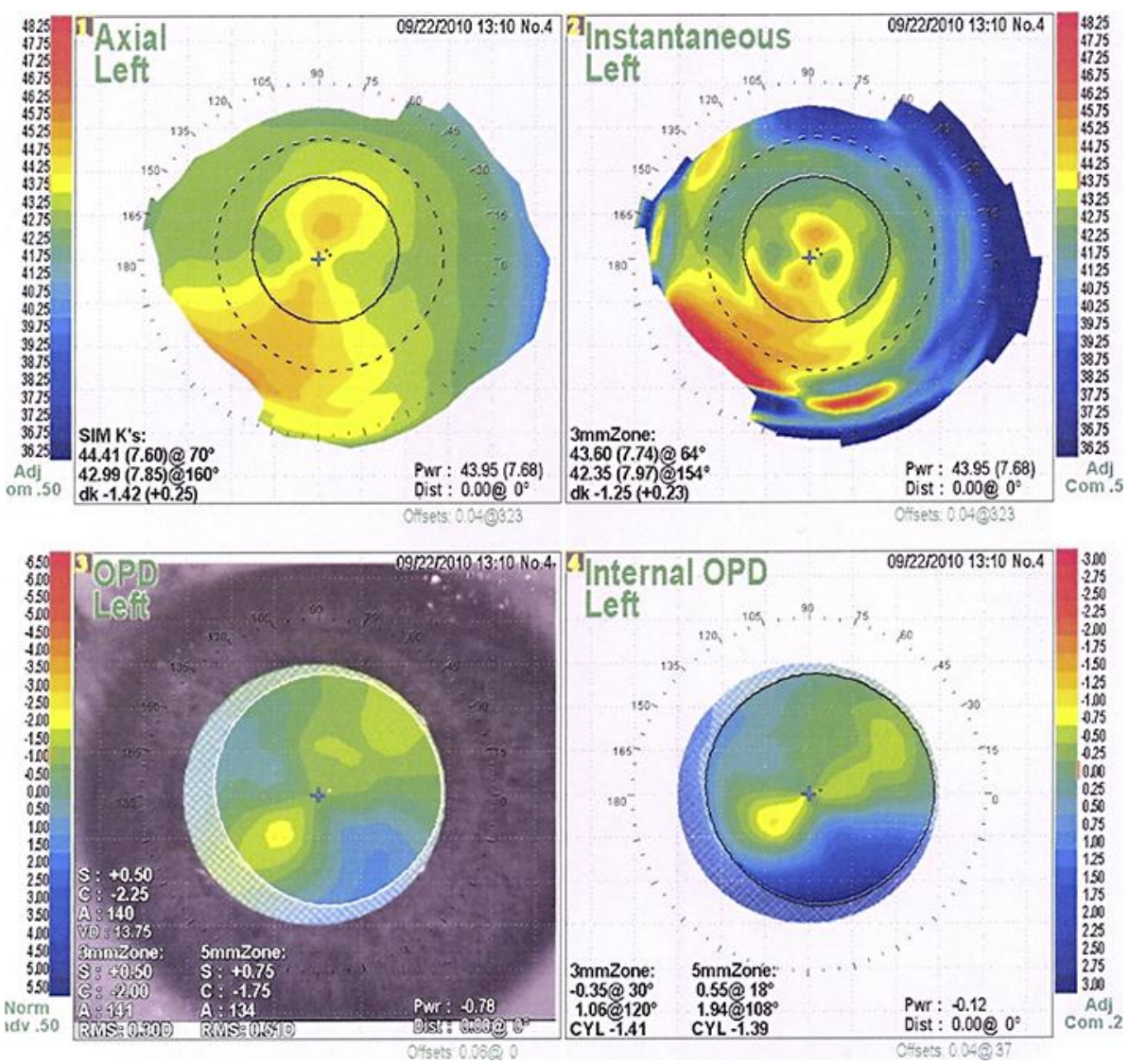

Fig. 1. OPD-Scan refractive power/corneal analyzer system printout shows internal astigmatism due to rotated toric IOL in the left eye. 
Case Reports in

Ophthalmology
Case Rep Ophthalmol 2013;4:138-143

DOI: $10.1159 / 000355415$

Gimbel et al.: Reverse Optic Capture to Stabilize a Toric Intraocular Lens

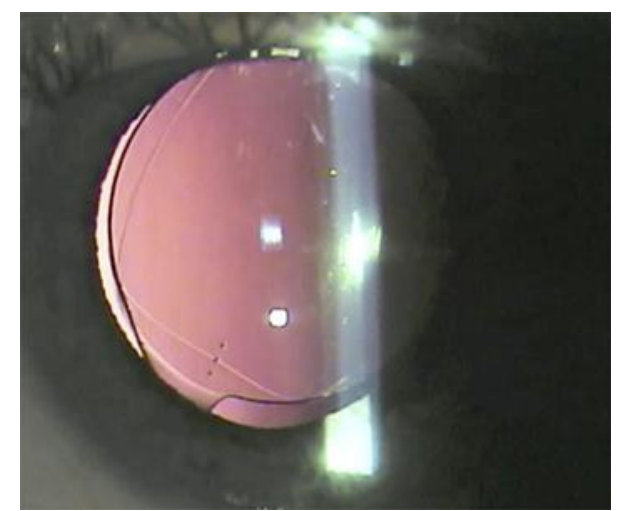

Fig. 2. Slit lamp image on the first postoperative day shows ROC in the left eye. 